\title{
Lung clearance index - useful parameter for assessing lung function in cystic fibrosis children
}

\author{
Mihaela Dediu', Ioana Mihaiela Ciuca', Voicu Tudorache², Liviu Laurentiu Pop ${ }^{1}$ \\ ${ }^{1}$ Pediatric Department, "Victor Babes" University of Medicine and Pharmacy, Timisoara, Romania \\ 2Pulmology Departament, "Victor Babes" University of Medicine and Pharmacy, Timisoara, Romania
}

\begin{abstract}
CF is a polymorphic disease whose evolution is marked by pulmonary pathology. Distal obstruction, demonstrated anatomopathological, can be evidenced by spirometry, which can only be used in certain age groups. Early detection of obstruction with appropriate therapy is essential to prolong the life expectancy of these patients. A promising parameter has been described relatively recently, lung clearance index obtained by the multiple wash-out technique. Several studies have demonstrated its efficacy and correlation with pulmonary morphological changes, pointing out that $\mathrm{LCl}$ is a feasible and specific parameter to detect small-age lung changes in the CF patient. In conclusion, lung clearance index is a specific parameter for the detection of cystic fibrosis pneumopathy and is currently the standard way to monitor CF pneumopathy, and in future to be used in the current practice of CF centers.
\end{abstract}

Keywords: lung clearance index, cystic fibrosis, lung function

\section{INTRODUCTION}

Cystic fibrosis (CF), clinically characterized by impressive clinical signs with pulmonary and gastrointestinal symptomatology, is the most common monogenic disease in the Caucasian population(1). Although the life expectancy of CF patients has increased in recent years, the mortality rate is almost constant, mainly due to the progressive degradation of pulmonary function $(1,2)$. Although the molecular basis of this disease (the CFTR gene) is currently known, researches conducted so far had not found effective curative therapy, prognosis and disease progression being dictated by lung status (2).

Mutations of transmembrane cystic fibrosis conductance regulatory gene (CFTR) cause decreased water volume and increased mucus viscosity by poorly delivering $\mathrm{Cl}^{-}$and $\mathrm{HCO}_{3}$.(2) Mucus stagnation is a favorable environment for development of viral and bacterial infections, chronic progressive inflammation, bronchiectasis's occurrence and lung's function alteration $(2,3)$.

\section{LUNG DISEASE IN CYSTIC FIBROSIS}

There are still many debates about when and how the first pulmonary changes occur, especially because of the heterogeneous nature of lung damage in this population. Animal models suggest that structural abnormalities of CF can occur in utero (1). It can be argued that the disease starts from the moment of conception when the sperm fertilizes the ovum to create an embryo with two pathological CFTR mutations (2).

Pulmonary function assessment is an essential part of clinical care for detecting changes in the lung and establishing appropriate treatment $(1,2)$, because the lung disease is the most influent feature for the global CF outcome (1). 
Obstruction of the airway is progressive, as demonstrated by spirometry, but pulmonary function can be preserved in normal parameters, both during childhood and adulthood $(3,4)$ by addressing multidisciplinary care and early structural changes detection.

Functional assessment of lung status is not representative for structural or anatomical damage of the lung. There is strong evidence that the progression of bronchiectasis may not be detected by spirometry for many years (5), whereas $30 \%$ of $\mathrm{CF}$ patients with normal spirometry already have structural damage to high resolution computed tomography $(\operatorname{HRCT})(6,7)$.

\section{LUNG CLEARANCE INDEX}

Multiple pulmonary functional tests for awake children with pre-school age have been proposed. These include: standard spirometry, forced oscillometry, plethysmography air flow resistance, residual functional capacity by gas dilution techniques and gas mixture index (8).

In cystic fibrosis patients, lung clearance index (LCI) is currently used in specialized centers, a parameter that reflects the degree of homogeneity of the lung and is measured by the multiple breath wash-out technique with gas mixing $(\mathrm{N} 2, \mathrm{O} 2, \mathrm{H} 2)$ $(7,8,9)$.

Recent studies have shown that LCI is a feasible and specific parameter to detect lung changes in pre-school, scholar and adult patients with CF. It is easier to achieve, even in younger children who do not cooperate performing spirometry as it is determined during tidal breath $(7,9)$.

Pathological processes that affect the peripheral airway will cause an increase in pulmonary diffusion heterogeneity (8). Ventilation's inequality affects the overall efficiency of gas mixing at lung level and can be measured by monitoring the washout of a control gas during tidal breathing. In a pathological process, this elimination process will have a longer duration, requiring more breathing (9).

Any wash-out gas test has a dispersion phase and a gas removal phase (7). In the case of extrinsic inert gases or non-residual pulmonary gases, during the dispersion phase, the marker gas is distributed at a known concentration (10). The dispersion process is completed when the concentration of the wash-out gas reaches the concentration of the dispersed gas. In the case of intrinsic inert gases, there is no dispersion phase and only a few tidal breaths are required to ensure that the nitrogen concentration (N2) is stable (80\%) (11).

Lung clearance index is a parameter that is obtained during the wash-out phase. In this phase, the subject inspires a gas that does not contain the marker gas (room air for hexafluoride sulfide, 100\% O2 for N2) (10). The marker gas should be inert and not be absorbed or excreted in the body. With each successive respiration of washing, a decrease in the maximum expired marker concentration is recorded $(7,9,11)$.

LCI has a numerical value that represents the number of tidal washing / breathing required to remove an amount of N2 up to the acceptable limit value(). The higher the degree of lung damage and the longer period the patient requires to reach the minimum level of acceptable N2 concentration, the higher LCI $(8,9)$.

LCI decreases during the infancy and the first childhood, then continues to maintain constantly until adulthood and increases slightly in old age (10). LCI value does not change with patient's height and is not dependent on patient's sex, which is particularly important in longitudinal studies in children (8).

Currently, LCI's determination is a technique performed in a few specialized centers and is often used for scientific purposes (11).

\section{ADVANTAGES AND DISADVANTAGES}

LCI's sensitivity to pulmonary disease in CF may reveal the underlying lung pathology, like small airway inflammation and obstruction (9), which is important in early detection of small's airway lesions and can be used for establishing a relation between the morphological lung disease and its functionality (12). Because does not necessitate a special breathing pattern like spirometry, being performed during normal tidal breathing, the lung clearance index is the ideal test for children due to relatively simple technique, requiring only tidal breathing, which can also be performed by infants $(13,14)$. It does not require cooperation or sedation and can be performed in children from 0 to 18 years of age, like many studies showed $(9,13)$.

Although LCI's increased sensitivity makes it a useful test for diagnosing early lung lesions, LCI does not provide important information for patients with severe obstructive lesions (8). LCI's determination is the only way to assess lung function in 
infants and small children, with a good sensitivity and specificity for cystic fibrosis's pneumopathy $(9,10,11)$.

\section{LUNG CLEARANCE INDEX IN CLINICAL PRACTICE}

Numerous studies have demonstrated the utility of LCI in detecting impaired's lung function (14), more easily than spirometry in pre - school and school - aged (15) children also in older patients (16). A study performed on 142 patients with CF, based on lung function assessment in Sweden, showed that LCI's value is the first to change before $\mathrm{FEF}_{50 \%}$ and $\mathrm{FVC}$, and FEV1 is the last parameter modified (17). Moreover, the same study highlighted the continued growth of LCI, along with pulmonary hyperinflation and "captive" air volume, after the age of 12 , when the $\mathrm{FEV}_{1}$ 's $\mathrm{Z}$ score stays constant.

LCI has also been reported in studies involving all age groups, from infants $(14)$ to adults $(13,18)$, making it particularly suited to long-term studies. Cystic fibrosis has an increased need for selecting sensitive and repeatable tests for therapeutic interventions (19); LCI has been proposed as an index to be followed in future studies on gene therapy in cystic fibrosis (20).

It has been used in two clinical trials to examine the clinical response to intravenous antibiotic therapy in children with $\mathrm{CF}$, during acute pulmonary exacerbations (18), being a useful and accurate parameter for evaluation of the outcome (22). Studies revealed a very good correlation between the ventilator inhomogeneity and length of the respiration expressed by LCI and the cough index and noctur- nal saturation (23), showing that longer the time necessary for the N2 washout, the more pronounced was the cough episodes and night desaturation. For a correct determination of reliable LCI , two technically acceptable N2 MBW might be necessary in school children for the evaluation of the ventilation, although is time consuming and longer (24) in order to achieve a good reliability and sensitivity (25).

\section{CONCLUSIONS}

In conclusion, lung clearance index is a specific parameter for the detection of early lung disease in cystic fibrosis, as well as for monitoring the pulmonary disease`s outcome, representing currently the standard parameter for monitoring pulmonary function in children with cystic fibrosis; as primary finding of pulmonary changes, followed by intensive and appropriated care will prolong the CF children life hope. LCI is nowadays mainly used in specialized CF centers, in our country at the National Cystic Fibrosis Centre Timisoara for 3 years and will be widely requested as a gold standard to be used in the current practice of CF specialized centers in near future. Lung clearance index is the standard parameter in $\mathrm{CF}$ lung research and its clinical utility has been clearly proven and sustained by multiple studies, achieving a goal of decades, who asked for a consistent, reproducible and correct endpoint indicative of cystic fibrosis prognosis. LCI has been advocate for various respiratory pathologies which implies small airways and its future and current use includes asthma, recurrent wheeze, primary ciliary dyskinesia therefore is expectation for a commonly forthcoming routine.

Conflict of interest: none declared Financial support: none declared

\section{REFERENCES}

1. Lahiri T et al. Clinical Practice Guidelines from the Cystic Fibrosis Foundation for Preschoolers with Cystic Fibrosis, Pediatrics. 2016 April; 137(4), 2015-1784

2. Suri R, Metcalfe $C$, Lees $B$ et al. Comparison of hypertonic saline and alternate-day or daily recombinant human deoxyribonuclease in children with cystic fibrosis: A randomised trial. Lancet. 2001; 358(9290):1316-21.

3. Marshall BC, Nelson EC. Accelerating implementation of biomedical research advances: Critical elements of a successful 10 year Cystic Fibrosis Foundation healthcare delivery improvement initiative. BMJ Qual Saf. 2014;23(SUPPL1).

4. CFF. Annual Report 2012. Cyst Fibros Found Patient Regist. 2012.

5. Mott LS, Park J, Murray CP et al. Progression of early structural lung disease in young children with cystic fibrosis assessed using CT. Thorax. 2012;67(6):509-16.

6. Lindblad A, Rubin L, Hop WCJ et al. Progression of lung disease on computed tomography and pulmonary function tests in children and adults with cystic fibrosis. Thorax. 2006;61(April 2004):80-5.
7. Gustafsson PM, De Jong PA, Tiddens HAWM et al. Multiple-breath inert gas washout and spirometry versus structural lung disease in cystic fibrosis. Thorax. 2008;63(2):129-34. An official American Thoracic Society/European Respiratory Society statement: Pulmonary function testing in preschool children. Am J Respir Crit Care Med. Jun 15 2007; 175: 1304-1345

9. Horsley A. Lung clearance index in the assessment of airways disease. Respir Med. 2009;103(6):793-9.

10. Subbarao P, Milla $C$, Aurora $P$ et al. Multiple-breath washout as a lung function test in cystic fibrosis: A cystic fibrosis foundation workshop report. Ann Am Thorac Soc. 2015;12(6):932-9.

11. Lum S, Stocks J., Stanojevic S. et al. Age and height dependence of lung clearance index and functional residual capacity. Eur Respir J. 2013;41(6):1371-7.

12. Ciuca IM, Dediu M, Tamas $L$ et al. Lung ultrasound score and the relation with lung clearance index, Journal of Cystic Fibrosis, Volume 17, Supplement 3, 2018, Pages S11-S12, ISSN 1569-1993
8. Beydon N, Davis SD, Lombardi E, Allen JL, Arets HG, Aurora P et al. 
13. Bedrossian CWM, Donald Greenberg S, Singer DB et al. The Lung in Cystic Fibrosis: A Quantitative Study Including Prevalence of Pathologic Findings Among Different Age Groups. Hum Pathol. 1976;7(2):195-204.

14. Lum S, Gustafsson $\mathrm{P}$, Ljungberg $\mathrm{H}$ et al. Early detection of cystic fibrosis lung disease: Multiple-breath washout versus raised volume tests. Thorax. 2007;62(4):341-7.

15. Aurora P, Bush A, Gustafsson P et al. Multiple-breath washout as a marker of lung disease in preschool children with cystic fibrosis. Am J Respir Crit Care Med. 2005;171(3):249-56.

16. Gustafsson PM, Aurora P, Lindblad A. Evaluation of ventilation maldistribution as an early indicator of lung disease in children with cystic fibrosis. Eur Respir J. 2003;22(6):972-9.

17. Ciuca IM, Dediu M, Vlad D et al. Lung Clearance Index and vitamin D. Journal of Cystic Fibrosis, Volume 17, Supplement 3, 2018, Page S94

18. Kraemer R, Blum A, Schibler A et al. Ventilation inhomogeneities in relation to standard lung function in patients with cystic fibrosis. Am J Respir Crit Care Med. 2005;171(4):371-8.

19. Horsley AR, Gustafsson PM, Macleod KA et al. Lung clearance index is a sensitive, repeatable and practical measure of airways disease in adults with cystic fibrosis. Thorax. 2008;63(2):135-40.
20. Rosenfeld M. An overview of endpoints for cystic fibrosis clinical trials: One size does not fit all. Proc Am Thorac Soc. 2007;4(4):299-301.

21. Davies JC, Cunningham S, Alton EWFW et al. Lung clearance index in CF: A sensitive marker of lung disease severity. Thorax. 2008;63(2):96-7.

22. Robinson PD et al. Using index of ventilation to assess response to treatment for acute pulmonary exacerbation in children with cystic fibrosis. Pediatr Pulmonol. 2009;44(8):733-42.

23. Bakker EM et al. Determining presence of lung disease in young children with cystic fibrosis: Lung clearance index, oxygen saturation and cough frequency. Journal of Cystic Fibrosis, Volume 11(3) 223 230

24. Green, Kent \& S Ejlertsen, Jacob \& Ring, Astrid \& Buchvald, Frederik \& Kongstad, Thomas \& Kobbernagel, Helene \& M Gustafsson, Per \& Nielsen, Kim. Abbreviation Modalities of Nitrogen Multiple-Breath Washout Tests in School Children With Obstructed Lung Disease. Pediatric Pulmonology (2015). 51. 10.1002/ppul.23339.

25. Horsley AR, Macleod KA, Robson AG, Lenney J, Bell NJ, Cunningham $S$ et al. Effects of cystic fibrosis lung disease on gas mixing indices derived from alveolar slope analysis. Respir Physiol Neurobiol. 2008;162(3):197-203 